\title{
Finite-time Flocking Control of A Swarm of Cucker-Smale Agents with Collision Avoidance
}

\author{
Jing Ma and Edmund M-K Lai \\ Department of Information Technology \& Software Engineering \\ Auckland University of Technology, Auckland, New Zealand \\ \{jing.ma,edmund.lai\}@aut.ac.nz
}

\begin{abstract}
This paper considers the application of finite-time control to a Cucker-Smale flocking model of autonomous agents with collision avoidance. A mathematical expression for the upper bound on the flocking time is derived. Previous results without considering collision avoidance showed that the flocking time decreases as the number of robots in the flock increases, which is counter-intuitive. We showed that, with collision avoidance, the flocking time indeed increases with the flock size. Our mathematical results are verified by computer simulation. Simulation results also show that certain control parameters and noise can be used to reduce the flocking time.

Index Terms-Finite-time, Cucker-Smale Model, Flocking, Col-
\end{abstract} lision Avoidance

\section{INTRODUCTION}

Swarm robotics is concerned with the design and deployment of a large group of autonomous robots (or agents) to achieve certain tasks collectively [1]-[3]. The coordination of such a robot swarm is typically inspired by the emergent collective behaviours observed in nature. An important emergent collective behaviours is flocking, where robots selforganized into an ordered motion (velocity alignment) from a disordered initial state. Several models of flocking exist that provide us with some understanding of this emergent behaviour [4]-[6]. In [5], a deterministic model is proposed which consists of three simple steering rules: separation, alignment and cohesion. Since it is a computational model rather than mathematical model, it has been successfully applied to simulation of flocking behaviour in computer animation. Its disadvantage is that it is not amenable to mathematical analyses.

A popular model of alignment is proposed in [6], commonly known as the Vicsek model. It consists of point-like selfpropelled particles that move at the same speed but in a random direction initially. Each particle updates the direction of its velocity by averaging those of its neighbours within a certain distance. It has been shown that a phase transition could occur and the particles become aligned and move in the same direction after some time. A mathematical proof of convergence is difficult, and often relies on assumptions on the way convergence. Cucker and Smale [4] then proposed a flocking model based on which asymptotic convergence is guaranteed when certain initial conditions are satisfied. It is commonly referred to as the Cucker-Smale model and has the added advantage of being a continuous-time model. For a group of $N$ autonomous robots, the state of robot $i$
$(1 \leq i \leq N)$, is described by its position $p_{i}$ and velocity $v_{i}$ in the following way:

$$
\left\{\begin{array}{l}
\dot{p_{i}}=v_{i} \\
\dot{v_{i}}=\frac{1}{N} \sum_{j=1}^{N} \psi\left(\left\|p_{j}-p_{i}\right\|\right)\left(v_{j}-v_{i}\right)
\end{array}\right.
$$

where $\psi$ is a positive decreasing function of the Euclidean distance between two robots. It is commonly known as the communication rate function and is given by

$$
\psi\left(\left\|p_{j}-p_{i}\right\|\right)=\frac{1}{\left(1+\left\|p_{j}-p_{i}\right\|^{2}\right)^{\beta}}
$$

It quantifies the influence of robot $j$ on the velocity of robot $i$. It has been proven mathematically in [4] that flocking will emerge if $\beta<1 / 2$. For $\beta \geq 1 / 2$, however, flocking is conditional on the initial state of the system. Both the Vicsek and the original Cucker-Smale models do not consider collision avoidance which is important for practical applications. Recently, the Cucker-Smale model has been extended by including a repelling force between robots to the original model to maintain a minimum distance between robots to avoid collision [7].

While these asymptotic convergence results are useful, in practical applications, it is desirable to ensure that flocking occurs within a finite time. Finite-time control was first considered in [8]. Since then, it has been studied from several different perspectives. In [9], a class of output feedback controllers that can achieve global finite-time stability for the double integrator system are proposed. Finite-time consensus algorithms have been developed for second-order [10], [11] as well as nonlinear agent dynamics [12]. Consensus with fixed and switching undirected topologies was considered in [13]. The authors showed that when a particular type of nonlinear inter-agent interaction is used, consensus can be reached in finite time. Distributed finite-time tracking and containment control are investigated in [14]-[17]. A distributed finitetime tracking protocol has been designed for networks with Euler-Lagrange agent dynamics [17]. It should be pointed out that finite-time convergence cannot take place in Lipschitz continuous systems. This is why the control actions have to be either discontinuous, non-smooth or non-Lipschitz continuous.

Recently, finite-time control theory has been applied to the Cucker-Smale model. In [18], [19], the original Cucker-Smale model has been modified to be non-Lipschitz continuous so 
that a finite upper bound on the flocking time could be established. An interesting result is that this flocking time bound decreases as the diameter of the flock $N$ increases. This seems to imply that if we want to lower the maximum flocking time, all we need to do is to increase the number of robots. But this phenomenon is caused by the fact that the model does not consider collisions of the robots. In practice, increasing the size of the flock will increase the chance of collision and so the flocking time should be higher than the above theoretical bound.

The objective of this paper is to establish an upper bound on the flocking time of a swarm of Cucker-Smale agents with collision avoidance using finite-time control. This theoretic result is then verified by computer simulation. Furthermore, we studied the effects of various finite-time control parameters on the flocking time as well as the flock diameter. We also studied the effect of uncertainty in the velocity estimates on the flocking time.

A brief introduction to finite-time control and a definition of flocking are presented in Section II. This is followed by a description of the finite-time Cucker-Smale model with collision avoidance that is studied in this paper. In Section IV, we present the mathematical proof that velocity consensus can be achieved in finite-time using this model and obtained a mathematical expression for an upper bound to the flocking time. Computer simulation results are presented in Section V.

\section{PREliminaries}

\section{A. Definition of Flocking}

Flocking is said to be achieved when the following two conditions are satisfied for a group of robots. It has been defined a very similar way in [18], where the difference of all velocities tends to zero in finite time and the diameter of the group is bounded. To investigate the control of flock diameter, the flocking would be defined as follows:

1) The velocity of every robot is virtually the same, i.e. for an arbitrarily small $\delta>0$,

$$
\left|v_{i}-v_{j}\right| \leq \delta
$$

for all $i, j \in[1, N], i \neq j$. The flocking time is defined as $T_{f}=\inf \left\{T:\left|v_{i}-v_{j}\right| \leq \delta\right\}$.

A useful measure is the average normalized velocity which is defined as [6]

$$
v_{a}=\frac{\left|\sum_{i=1}^{N} v_{i}\right|}{\sum_{i=1}^{N}\left|v_{i}\right|}
$$

The above condition is equivalent to $\left|1-v_{a}\right|<\delta^{\prime}$ for some small $\delta^{\prime}>0$.

2) The distance between any two robots is bounded by a positive finite constant $\epsilon$ which we shall refer to as the flock diameter. That is,

$$
0<\sup _{t \geq T_{f}}\left\|p_{i}-p_{j}\right\|<\epsilon
$$

for all $1 \leq i, j \leq N$.

\section{B. Finite-time Control Theory}

It has been known that bang-bang control can be used to drive a feedback control system to its equilibrium point in a time-optimal manner [20]. However, the discontinuous control law could cause problems such as chattering [21]. To overcome this problem, time-optimal continuous and bounded controllers were first considered in [8]. The author proposed first and second order dynamic equations for the controller that can be shown to reach equilibrium within a finite time, as opposed to asymptotic convergence of conventional controllers. These controllers are known as finite-time controllers.

Finite-time control was later put on a more rigorous theoretical footing by [22]. The authors showed that the control law

$$
u=-l_{1} \operatorname{sgn}\left(x_{1}\right)\left|x_{1}\right|^{\alpha_{1}}-l_{2} \operatorname{sgn}\left(x_{2}\right)\left|x_{2}\right|^{\alpha_{2}}
$$

where

$$
\begin{array}{r}
0<\alpha_{1}<1, \quad \alpha_{2}=\frac{2 \alpha_{1}}{1+\alpha_{1}}, \\
\operatorname{sgn}(s)= \begin{cases}1, & s>0 \\
0, & s=0 \\
-1, & s<0\end{cases}
\end{array}
$$

and $l_{1}, l_{2}>0$ are able to stabilize in finite-time a double integrator system with dynamics described by

$$
\dot{x}_{1}=x_{2}, \quad \dot{x}_{2}=u \text {. }
$$

This control law is a homogeneous function of degree $\alpha_{2}$ and it is continuous. This dynamical system has been shown to be globally finite-time stable. Most significantly, by using Lyapunov stability theory, the authors were able to derive an upper bound for the settling time.

The convergence time is obtained in [25] using the following lemma. Our proof of the convergence time for the CuckerSmale model with colllision avoidance in Section III is also based on the same lemma.

Lemma 1: Assume that a continuous, positive-definite function $V(t)$ satisfies the following differential inequality:

$$
\dot{V}(t) \leq-k V^{\rho}(t)
$$

where $\mathrm{k}, \rho$ are two constants. Then, for any given $t_{0}, V(t)$ satisfies the following inequality:

$$
V^{1-\rho}(t) \leq V^{1-\rho}\left(t_{0}\right)-k(1-\rho)\left(t-t_{0}\right), \quad t_{0} \leq t \leq t_{1},
$$

and $V(t) \equiv 0, t \geq t_{1}$ with $t_{1}$ is given by $t_{1}=t_{0}+$ $\left[V\left(t_{0}\right)^{1-\rho}\right] / k(1-\rho)$.

\section{Finite-time CuCKeR-Smale Model With Collision AVOidance}

One way to prevent robots in a swarm from colliding is to introduce a repelling force between robots. This repelling force should come into effect when two robots are too close to each other. Let $d_{0}>0$ be the minimum distance between any two robots such that if $\left\|p_{j}-p_{i}\right\| \leq d_{0}$, then we consider that robots $i$ and $j$ have collided. A repelling force function can be used. In [24], it has been suggested that this repelling 
force function $f:\left(d_{0}, \infty\right] \rightarrow[0, \infty)$ should have the following properties:

$$
\left\{\begin{array}{l}
\int_{d_{0}}^{d_{1}} f(r) d r=\infty \\
\int_{d_{1}}^{\infty} f(r) d r<\infty
\end{array}\right.
$$

for some $d_{1}>d_{0}$. By incorporate this function into (1), we have a new Cucker-Smale system with collision avoidance:

$$
\left\{\begin{aligned}
\dot{p}_{i}= & v_{i} \\
\dot{v}_{i}= & \frac{1}{N} \sum_{j=1}^{N} \psi\left(\left\|p_{j}-p_{i}\right\|\right)\left(v_{j}-v_{i}\right) \\
& +\frac{1}{N} \sum_{j \neq i} f\left(\left\|p_{j}-p_{i}\right\|^{2}\right)\left(p_{j}-p_{i}\right)
\end{aligned}\right.
$$

for $1 \leq i \leq N$. Here, $f(\cdot)$ is the repelling function.

Finite-time control (6) can now be applied to (11). In this case, $p_{i}$ and $v_{i}$ are $x_{1}$ and $x_{2}$ in (6) respectively. The finitetime controlled Cucker-Smale model with collision avoidance is therefore given by:

$$
\left\{\begin{aligned}
\dot{p}_{i}= & v_{i} \\
\dot{v}_{i}= & \frac{1}{N} \sum_{j=1}^{N} \psi\left(\left\|p_{j}-p_{i}\right\|\right) \operatorname{sgn}\left(v_{j}-v_{i}\right)^{\theta} \\
& -\frac{1}{N} l_{1} \sum_{j \neq i} f\left(\left\|p_{j}-p_{i}\right\|^{2}\right) \operatorname{sgn}\left(v_{j}-v_{i}\right)^{\alpha_{1}} \\
& -\frac{1}{N} l_{2} \sum_{j \neq i} f\left(\left\|p_{j}-p_{i}\right\|^{2}\right) \operatorname{sgn}\left(p_{i}-p_{j}\right)^{\alpha_{2}}
\end{aligned}\right.
$$

for $1 \leq i \leq N$. Here, we used the notation

$$
\operatorname{sgn}(x)^{\alpha}=\operatorname{sgn}(x)|x|^{\alpha}
$$

where $\alpha \in R$. The finite-time control parameters must satisfy (7), and $0<\theta<1, l_{1}, l_{2}>0$.

There are three terms on the right-hand side of $\dot{v}_{i}$ in (12). The first one is to achieve finite-time flocking which is similar to [18], [19]. The other two terms are utilized to adjust velocities to ensure collision does not occur. We shall denote the finite-time convergence of each of these terms by $T_{0}, T_{1}$ and $T_{2}$ respectively.

\section{Flocking Time}

We shall now prove that the flocking time of the system given by (12) is finite.

Theorem 1: The velocities of the autonomous robots in the system given by (12) converges to the same velocity in a finite amount of time with an upper bound $T_{f}$ given by

$$
T_{f} \leq \max \left\{T_{0}, T_{1}, T_{2}\right\}
$$

where

$$
\begin{aligned}
& T_{0}=C_{0} N^{-\frac{\theta+1}{2}}, \quad C_{0}=\frac{2 V(0)^{\frac{1-\theta}{2}}}{\psi^{*} \sqrt{2}^{\theta+1}(1-\theta)} \\
& T_{1}=C_{1} N^{-\frac{\alpha_{1}}{2}}, \quad C_{1}=\frac{4 V(0)^{1-\frac{\alpha_{1}}{2}}}{l_{1} M \sqrt{2}^{\alpha_{1}}\left(2-\alpha_{1}\right)} \\
& T_{2}=C_{2} N^{-\frac{\alpha_{2}}{2}}, \quad C_{2}=\frac{4 V(0)^{1-\frac{\alpha_{2}}{2}}}{l_{2} M \sqrt{2}^{\alpha_{2}}\left(2-\alpha_{2}\right)} \\
& V(0)=\sum_{i=1}^{N}\left\|v_{i}(0)\right\|^{2}
\end{aligned}
$$

$T_{0}$ is to achieve flocking behaviour, and it has been proven in [18], [19], where the communication rate $\psi$ from 2 satisfies a lower bound conditions. In other words, there exists $\psi^{*}>0$ such that $i n f_{s \geq 0} \psi(s) \geq \psi^{*} . T_{1}$ and $T_{2}$ are to avoid collision that would be proved in this paper.

Proof: From [18], [19], proving the finite-time convergence of velocity is the same as proving $V(t)$ tends to zero in finite time. Applying finite-time control (6) to (12), we have

$$
\begin{aligned}
\dot{V}(t) & =\frac{2}{N} \sum_{i=1}^{N} \sum_{j=1}^{N} v_{i} \psi\left(\left\|p_{j}-p_{i}\right\|\right) \operatorname{sgn}\left(v_{j}-v_{i}\right)^{\theta} \\
& -\frac{1}{N} l_{1} \sum_{i=1}^{N} \sum_{j \neq i} f\left(\left\|p_{j}-p_{i}\right\|^{2}\right) \operatorname{sgn}\left(v_{j}-v_{i}\right)^{\alpha_{1}} \\
& -\frac{1}{N} l_{2} \sum_{i=1}^{N} \sum_{j \neq i} f\left(\left\|p_{j}-p_{i}\right\|^{2}\right) \operatorname{sgn}\left(p_{i}-p_{j}\right)^{\alpha_{2}}
\end{aligned}
$$

It consists of three terms which we shall denote by $\dot{V}_{0}(t)$, $\dot{V}_{1}(t)$ and $\dot{V}_{2}(t)$ respectively.

It has been proven in [18], [19] that $\dot{V}_{0}(t)$ converges in finite time. Using Lemma $1, V_{i}(t) \equiv 0, i=1,2, \ldots, N$ for $t \geq T_{0}$ with $T_{0}$ given by (15). Now we shall deal with the terms $\dot{V}_{1}(t)$ and $\dot{V}_{2}(t)$ which are related to collision avoidance. Since $f$ is a non-increasing function according to (10), there exists a constant $M$ such that, for all $i \neq j$ and $t \in[0, T)$, $f\left(\left\|p_{j}-p_{i}\right\|^{2}\right) \leq M$ [24]. Thus,

$$
\begin{aligned}
\dot{V}_{1}(t) & =-l_{1} \sum_{i=1}^{N} \sum_{j \neq i} f\left(\left\|p_{j}-p_{i}\right\|^{2}\right) \operatorname{sgn}\left(v_{j}-v_{i}\right)\left|v_{j}-v_{i}\right|^{\alpha_{1}} \\
& \leq-\frac{1}{N} l_{1} \sum_{i=1}^{N} \sum_{j \neq i} f\left(\left\|p_{j}-p_{i}\right\|^{2}\right)\left|v_{j}-v_{i}\right|^{\alpha_{1}} \\
& \leq-\frac{1}{N} l_{1} M \sum_{i=1}^{N} \sum_{j \neq i}\left|v_{j}-v_{i}\right|^{\alpha_{1}} \\
& =-\frac{1}{N} l_{1} M \sum_{i=1}^{N} \sum_{j \neq i}\left(\left|v_{j}-v_{i}\right|^{2}\right)^{\frac{\alpha_{1}}{2}} \\
& \leq-\frac{1}{N} l_{1} M(2 N)^{\frac{\alpha_{1}}{2}} V(t)^{\frac{\alpha_{1}}{2}}
\end{aligned}
$$

We can apply Lemma 1 to $\dot{V}_{1}(t)$ similar to $\dot{V}_{0}(t)$ and hence $T_{1}$ is given by (15).

Applying the upper bound of $f$ to $\dot{V}_{2}(t)$ in a similar way, 
we obtain

$$
\begin{aligned}
\dot{V}_{2}(t)= & -\frac{1}{N} l_{2} \sum_{i=1}^{N} \sum_{j \neq i} f\left(\left\|p_{j}-p_{i}\right\|^{2}\right) \operatorname{sgn}\left(p_{i}-p_{j}\right)\left|p_{i}-p_{j}\right|^{\alpha_{2}} \\
\leq & -\frac{1}{N} l_{2} M(2 N)^{\frac{\alpha_{2}}{2}} \sum_{i=1}^{N} \sum_{j \neq i}\left|p_{i}-p_{j}\right|^{\alpha_{2}} \\
= & -\frac{1}{N} l_{2} M(2 N)^{\frac{\alpha_{2}}{2}} \sum_{i=1}^{N} \sum_{j \neq i}\left|\int_{0}^{t} v_{i}(t)-\int_{0}^{t} v_{j}(t)\right|^{\alpha_{2}} \\
= & -\frac{1}{N} l_{2} M(2 N)^{\frac{\alpha_{2}}{2}} \sum_{i=1}^{N} \sum_{j \neq i} \mid\left(v_{i}(t)-v_{j}(t)\right) \\
& +\left.\left(v_{j}(0)-v_{i}(0)\right)\right|^{\alpha_{2}}
\end{aligned}
$$

Since $0<\alpha_{2}<1$,

$$
\begin{aligned}
\dot{V}_{2}(t) \leq & -\frac{1}{N} l_{2} M(2 N)^{\frac{\alpha_{2}}{2}} \sum_{i=1}^{N} \sum_{j \neq i}\left|v_{i}(t)-v_{j}(t)\right|^{\alpha_{2}} \\
& -\frac{1}{N} l_{2} M(2 N)^{\frac{\alpha_{2}}{2}} \sum_{i=1}^{N} \sum_{j \neq i}\left|v_{j}(0)-v_{i}(0)\right|^{\alpha_{2}} \\
= & -\frac{1}{N} l_{2} M(2 N)^{\frac{\alpha_{2}}{2}} V(t)^{\frac{\alpha_{2}}{2}} \\
& -\frac{1}{N} l_{2} M(2 N)^{\frac{\alpha_{2}}{2}} \sum_{i=1}^{N} \sum_{j \neq i}\left|v_{j}(0)-v_{i}(0)\right|^{\alpha_{2}} \\
\leq & -\frac{1}{N} l_{2} M(2 N)^{\frac{\alpha_{2}}{2}} V(t)^{\frac{\alpha_{2}}{2}}
\end{aligned}
$$

Therefore we can apply Lemma 1 again to obtain $T_{2}$ given in (15). Hence the theorem is proved.

In addition to alignment of velocity, flocking requires that the flock diameter be finite. We shall now demonstrate that $\mathrm{P}(\mathrm{t})$ is bounded [18]. We have

$$
\begin{aligned}
\dot{P}(t) & =2 \sum_{i=1}^{N}<p_{i}, v_{i}> \\
& \leq 2 \sum_{i=1}^{N}\left\|p_{i}\right\|\left\|v_{i}\right\| \\
& \leq 2 P^{\frac{1}{2}}(t) V^{\frac{1}{2}}(t)
\end{aligned}
$$

Assume that $P(t) \leq \Gamma(t)$, where $\Gamma(t)$ satisfies $\dot{\Gamma}(t)=$ $2 \Gamma^{\frac{1}{2}}(t) V^{\frac{1}{2}}(t)$ with $\Gamma(0)=P(0)$. Then we have

$$
\Gamma^{\frac{1}{2}}(t)=\Gamma^{\frac{1}{2}}(0)+\int_{0}^{t} V^{\frac{1}{2}}(s) d s
$$

and

$$
P^{\frac{1}{2}}(t)=P^{\frac{1}{2}}(0)+\int_{0}^{t} V^{\frac{1}{2}}(s) d s
$$

Since $V(t) \equiv 0$ after $t>T_{f}$,

$$
\begin{aligned}
P^{\frac{1}{2}}(t) & =P^{\frac{1}{2}}(0)+\int_{0}^{T_{f}} V^{\frac{1}{2}}(s) d s \\
& =P^{\frac{1}{2}}(0)+V(0)\left(T_{f}\right)<\infty
\end{aligned}
$$

Thus the robots are flocking after $T_{f}$.
TABLE I

FLOCK DIAMETER

\begin{tabular}{|c|c|c|}
\hline$N$ & $\begin{array}{c}\text { Flock Diameter } \\
\text { With Collision Avoidance }\end{array}$ & $\begin{array}{c}\text { Flock Diameter } \\
\text { Without Collision Avoidance }\end{array}$ \\
\hline 10 & 0.9611 & 0.8507 \\
\hline 15 & 0.9997 & 0.8960 \\
\hline 20 & 1.0259 & 0.9027 \\
\hline 25 & 1.0641 & 0.9082 \\
\hline 30 & 1.1328 & 0.9281 \\
\hline 35 & 1.1790 & 0.9466 \\
\hline 40 & 1.2277 & 0.9639 \\
\hline 45 & 1.2578 & 0.9845 \\
\hline 50 & 1.2843 & 1.0030 \\
\hline \hline
\end{tabular}

\section{Simulation Results}

The flocking time given by (14) will be compared with computer simulation results. We consider robots moving in an infinitely large two-dimensional space. Consequently, they will not encounter any boundaries. Each robot moves with the same speed but initially at a random direction. For simplicity, the communication rate $\psi$ is assumed to be 1 . This is consistent with previously published results in [18] and elsewhere.

For convenience, distances and time shall be dimensionless. Each robot moves with a constant speed of 0.5 per unit time. The initial direction is uniformly random in $[0,2 \pi)$. The distances $d_{1}$ and $d_{0}$ for the repelling force are set at 0.3 and 0.2 , respectively. The maximum value of repelling function is fixed at 5 . The system is considered to be in a flocking state when $v_{a} \geq 0.99$. The flock diameter is measured when this condition occurs. Each simulation scenario is repeated 20 times with different random initial values. The results presented are the average values.

\section{A. Flocking Time and Diameter}

First, we shall verify that the flocking time derived in Theorem 1 is correct using simulation. Apart from the parameters specified above, the remaining ones used in this part of the simulation are: $\theta=1 / 2, \alpha_{1}=1 / 2, \alpha_{2}=2 / 3$ and $l_{1}=1$, $l_{2}=1$. Figure 1 shows the flocking times computed from (14) for different values of $N$, and compared with the simulation results. The graph shows that the upper bound of the flocking time is correct. However, the bound is not particularly tight.

As the total number of robots $N$ increases, the flocking time increases significantly. Figure 2 shows the simulation results with and without collision avoidance. Without considering collision avoidance, as in [18], [19], flocking time decreases as $N$ increases. For instance, with $N=50$, the flocking time is approximately 1.43 with collision avoidance compared with less than 0.2 without collision avoidance. Thus the convergence results without considering collision are obviously over optimistic.

Table I shows the flock diameters when the velocities are aligned. The values indicate that the flock remains tight which indicates that flocking does occur. Flock diameters are slightly larger with repelling force in place. 


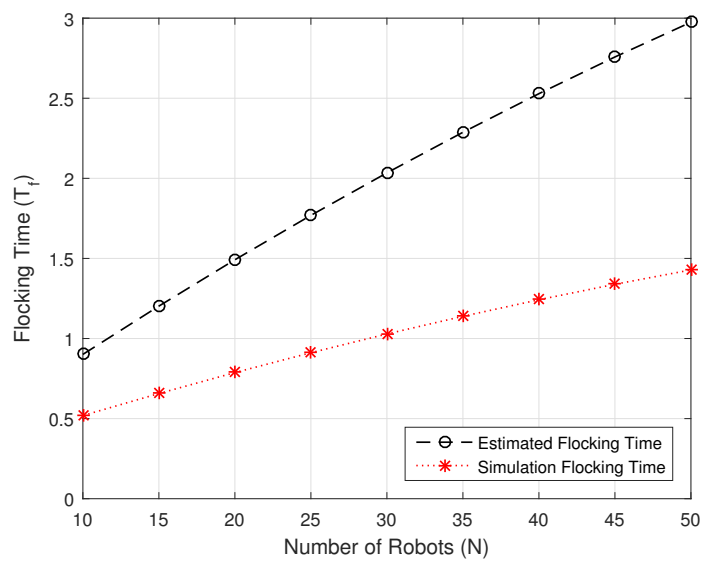

Fig. 1. Finite Flocking time of Cucker-Smale Model with Collision Avoidance

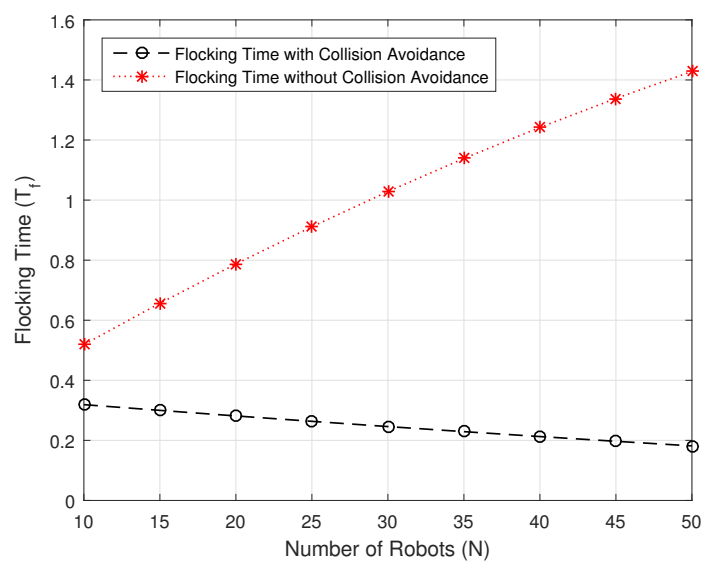

Fig. 2. Finite Flocking time of Cucker-Smale Model Depending on Collision Avoidance

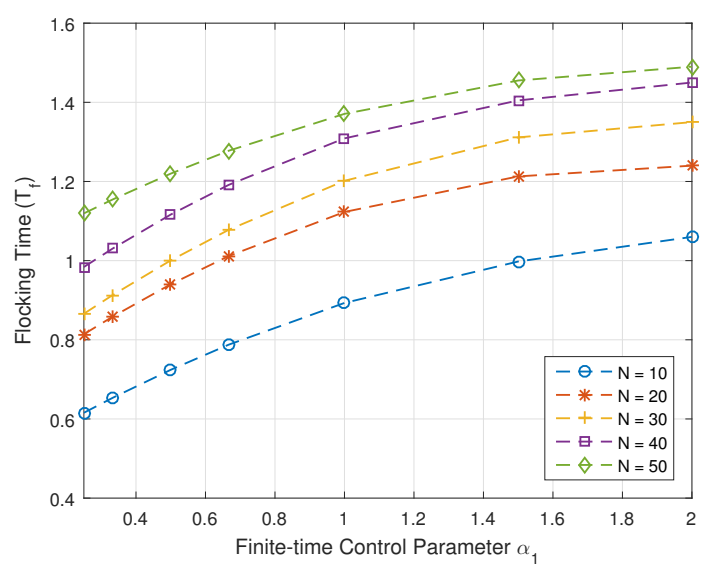

Fig. 3. Flocking Time of Different Control Coefficients

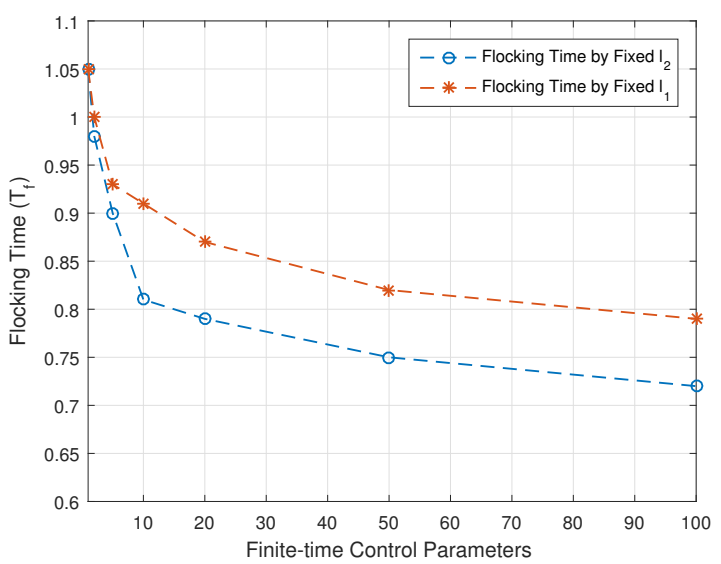

Fig. 4. Flocking Time of Different Control Parameters

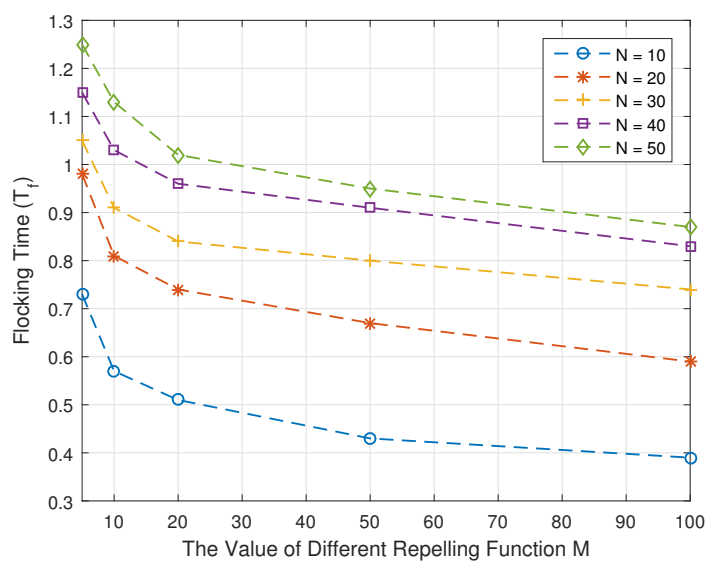

Fig. 5. Flocking Time of the Different Value of Repelling Function

\section{B. Effects of Flocking Control Parameters}

Next, we consider the effects of the flocking control parameters $\alpha_{1}, \alpha_{2}, l_{1}, l_{2}$ and $M$ on the flocking time. Figure 3 shows the effect of $\alpha_{1}$ and $\alpha_{2}$ on the flocking time for $\alpha_{1}=$ $\{1 / 4,1 / 3,1 / 2,2 / 3,1,3 / 2,2\}$. Here, $\theta=0.5, l_{1}=l_{2}=1$, $M=5$. Recall that $\alpha_{2}$ is related to $\alpha_{1}$ by $\alpha_{2}=\frac{2 \alpha_{1}}{1+\alpha_{1}}$. Other parameters are the same as in previous simulations. These results show that for all flock sizes considered, reducing $\alpha_{1}$ will result in a shorter flock time.

Figure 4 shows the effects of varying $l_{1}$ and $l_{2}$ In this case, we used $\alpha_{1}=1 / 2, \alpha_{2}=2 / 3, M=5$, and $N=30$. The blue curve shows the flocking time with $l_{2}=1$ and $l_{1}=$ $\{1,5,10,20,50,100\}$. The red curve has $l_{1}=1$ and $l_{2}$ varying instead. These results show that flocking time could be reduced by increasing $l_{1}$ and/or $l_{2}$. Increasing $l_{1}$ has a more pronounced effect on the flocking time compared with $l_{2}$.

Next, we consider the effects of strength of the repelling function. With $l_{1}=l_{2}=1, \alpha_{1}=1 / 2$ and $\alpha_{2}=2 / 3$, the effect on the flocking time for $M=\{5,10,20,50,100\}$ is shown in Figure 5. It clearly shows that the flocking time reduces as $M$ increases. But it is at the expense of a larger 


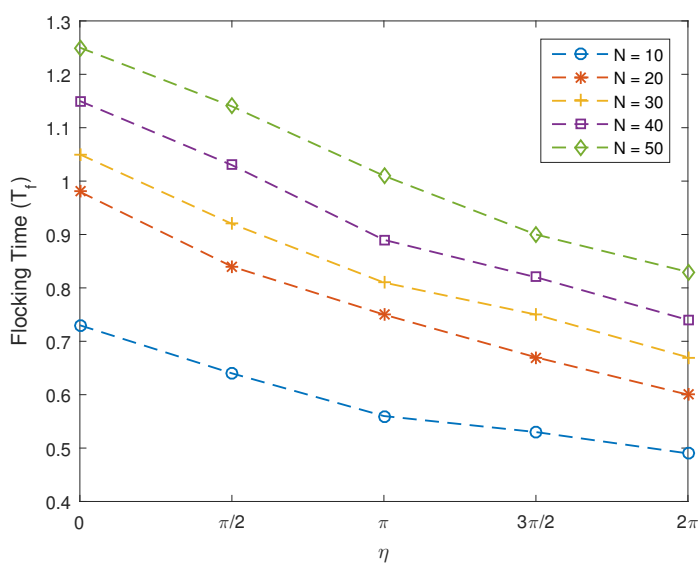

Fig. 6. Flocking Time of Different Noise

flock diameter.

Finally, we examine the effect of noise on the velocity adjustment for each robot similar to the way it is commonly done with the Vicsek model. The amount of noise is uniformly distributed between $[-\eta, \eta]$ where $\eta \in(0,2 \pi)$. Figure 6 shows that the flocking time decreases as noise is increased. Noise is particularly effective in reducing flocking time for larger flocks.

\section{Vi. Conclusions}

In this paper, finite-time control is applied to a CuckerSmale flocking model with collision avoidance. Convergence is mathematically proved and an upper bound of the flocking time is derived. The mathematical results have been verified by computer simulations. They show that flocking time increases with the flock size which is different from results reported previously for Cucker-Smale models without collision avoidance. Results also show that control parameters $l_{1}$ and $l_{2}$ as well as the repelling force strength and noise can be used to reduce the flocking time.

\section{REFERENCES}

[1] M. Dorigo, D. Floreano, L. M. Gambardella, F. Mondada, S. Nolfi, T. Baaboura,... and D. Burnier. Swarmanoid: a novel concept for the study of heterogeneous robotic swarms. IEEE Robotics \& Automation Magazine, 20(4):60-71, 2013.

[2] V. Kumar and F. Sahin. Cognitive maps in swarm robots for the mine detection application. IEEE International Conference on Systems, Man and Cybernetics, 4:3364-3369, 2003.

[3] D.P. Stormont. Autonomous rescue robot swarms for first responders. IEEE International Conference on Computational Intelligence for Homeland Security and Personal Safety, pages 151-157, 2005.

[4] F. Cucker and S. Smale. Emergent behaviour in flocks. IEEE Transactions on Automatic Control, 52(5):852-862, 2007.

[5] C. W. Reynolds. Flocks, Herds and Schools: A Distributed Behavioural Model. SIGGRAPH Comput. Graph., 21(4):25-34, 81987.

[6] T. Vicsek, A. Czirók, E. Ben-Jacob, I. Cohen, and O. Shochet. Novel type of phase transition in a system of self-driven particles. Physical Review Letters, 75(6):1226-1229, 1995.

[7] F. Cucker and J.-G. Dong. Avoiding collisions in flocks. IEEE Transactions on Automatic Control, 55(5):1238-1243, 2010.

[8] V. T. Haimo. Finite time controllers. SIAM Journal on Control and Optimization, 24(4):760-770, 1986.
[9] Y. Hong, J. Huang, and Y. Xu. On an output feedback finite-time stabilization problem. IEEE Transactions on Automatic Control, 46(2):305309, 2001.

[10] X. Wang and Y. Hong. Finite-time consensus for multi-agent networks with second-order agent dynamics. In Proceedings of the IFAC World Congress, pages 15185-15190, 2008.

[11] J. Cortés. Finite-time convergent gradient flows with applications to network consensus. Automatica, 42(11):1993-2000, 2006.

[12] Y. Cao, W. Ren, F. Chen, and G. Zong. Finite-time consensus of multiagent networks with inherent nonlinear dynamics under an undirected interaction graph. In American Control Conference (ACC), pages 40204025, 2011.

[13] F. Jiang and L. Wang. Finite-time information consensus for multi-agent systems with fixed and switching topologies. Physica D: Nonlinear Phenomena, 238(16):1550-1560, 2009.

[14] X. Wang, S. Li, and P. Shi. Distributed finite-time containment control for double-integrator multiagent systems. IEEE Transactions on Cybernetics, 44(9):1518-1528, 2014.

[15] X. He, Q. Wang, and W. Yu. Distributed finite-time containment control for second-order nonlinear multi-agent systems. Applied Mathematics and Computation, 268:509-521, 2015.

[16] D. Yu, Q. Wu, and L. Song. Finite time estimation and containment control of second order perturbed directed networks. In IEEE Conference on Decision and Control and European Control (CDC-ECC), pages 4126-4131, 2011.

[17] Y. Zhao, Z. Duan, and G. Wen. Distributed finite-time tracking of multiple Euler-Lagrange systems without velocity measurements. International Journal of Robust and Nonlinear Control, 2014.

[18] Y. Han, D. Zhao, and Y. Sun. Finite-time flocking problem of a cuckersmale-type self-propelled particle model. Complexity, 2015.

[19] S. Yongzheng, L. Feng, L. Wang, and S. Hongjun. Finite-time flocking of Cucker-Smale systems. In 34th Chinese Control Conference, pages 7016-7020, 2015.

[20] A. Bryson and Y. Ho. Applied Optimal Control. Wiley, 1975.

[21] I. Flugge-Lotz. Discontinuous and Optimal Control. McGraw-Hill, 1968.

[22] S. P. Bhat and D. S. Bernstein. Finite-time stability of continuous autonomous systems. SIAM Journal on Control and Optimization, 38(3):751-766, 2000.

[23] Y. Hong, Y. Xu, and J. Huang. Finite-time control for robot manipulators. Systems \& Control Letters, 46(4):243-253, 2002.

[24] F. Cucker and J.-G. Dong. A general collision-avoiding flocking framework. IEEE Transactions on Automatic Control, 56(5):1124-1129, 2011.

[25] H. Wang, Z. Han, and Q. Xie and W. Zhang. Finite-time synchronization of uncertain unified chaotic systems based on CLF. Nonlinear Analysis: Real World Applications, 10(5): 2842-2849, 2009. 